\title{
Cavernous Sinus Thrombophlebitis Related to Dental Infection
}

\section{-Two Case Reports-}

\author{
Hiroaki OKАмОтO, ${ }^{1}$ Atsushi Ogata, ${ }^{1}$ Masafumi Kosugi, ${ }^{2}$ \\ Hiroshi TAKASHIMA, ${ }^{2}$ Shuji SAKATA,${ }^{1}$ and Toshio MATSUSHIMA ${ }^{3}$ \\ Departments of ${ }^{1}$ Neurosurgery and ${ }^{2}$ Neurology, Saga Prefectural Hospital Koseikan, Saga, Saga; \\ ${ }^{3}$ Department of Neurosurgery, Saga University, Saga, Saga
}

\begin{abstract}
Two cases of cavernous sinus thrombophlebitis (CST) caused by dental infection are described. A 64year-old woman presented with palsies of the left oculomotor and trochlear nerves after tooth extraction for dental caries in the left maxilla. A 54-year-old man presented with palsy of the left trochlear nerve, sensory disturbance in the ophthalmic and maxillary divisions of the left trigeminal nerve, ptosis, proptosis, and chemosis after dental treatment for caries and periodontitis in the left maxilla. In both patients, computed tomography and magnetic resonance imaging with contrast medium showed nonenhanced lesions within the left cavernous sinus and dilation of the superior ophthalmic veins, which indicated CST. These conditions were resolved by administration of broad-spectrum antibiotics. CST is rare but lethal, so prompt diagnosis is crucial, and immediate appropriate treatment is essential.
\end{abstract}

Key words: cavernous sinus thrombophlebitis, cavernous sinus syndrome, dental infection, antibiotic therapy, anticoagulant therapy

\section{Introduction}

Cavernous sinus thrombophlebitis (CST) is fortunately now rare, since the mortality rate was estimated at approximately $100 \%$ before the introduction of antibiotics, but the prognosis has remarkably improved for patients with CST. ${ }^{1,3,7,9,10)}$ However, $13.6 \%$ mortality and $22.7 \%$ morbidity were reported in 28 CST cases in $1977,{ }^{10)}$ so CST is still a life-threatening disease unless the correct antibacterial treatment is provided. We report 2 cases of CST caused by dental infection. CST is rare, but should be strongly considered as a possible underlying disease in patients with cavernous sinus syndrome.

\section{Case Reports}

Case 1: A 64-year-old woman complained of headache 2 days after extraction of a canine tooth with caries in the left maxilla by a dentist. Non-steroidal anti-inflammatory drugs (NSAID) and antibiotics (cefcapene) were ineffective. She developed diplopia and left ptosis 8 days after tooth extraction. After consulting a neurologist at another hospital, she was diagnosed with left oculomotor palsy and was subsequently referred to our hospital.

On admission, the patient was alert and well oriented. Neurological examination revealed almost total external ophthalmoplegia (palsies of the oculomotor and trochlear nerves except for the abducens nerve), ptosis, and mydriasis on the left side. No proptosis, chemosis, or abnormalities of other cranial nerves were observed. She had no swelling of her face or oral cavity, and dental examination did not reveal any local inflammation such as abscess at the site of the tooth extraction. Her body temperature was $38^{\circ} \mathrm{C}$, and laboratory analysis showed a peripheral white blood cell (WBC) count of $12700 / \mu \mathrm{l}$ and C-reactive protein (CRP) level of $6.6 \mathrm{mg} / \mathrm{dl}$. Cerebrospinal fluid (CSF) from a lumbar puncture contained $30 \mathrm{WBCs} / \mathrm{mm}^{3}(66 \%$ neutrophils) with normal opening pressure, and normal protein and glucose levels. Blood and CSF culture findings were negative.

Head computed tomography (CT) did not show any abnormalities, but CT with contrast medium and magnetic resonance (MR) imaging with gadolinium revealed a nonenhanced lesion within the left cavernous sinus, indicating possible thrombus, and dilation of the bilateral superior ophthalmic veins (Figs. 1 and 2). These images did not suggest any orbital cellulitis or sinusitis. Cerebral angiography showed non-filling of the cavernous sinus and pooling of contrast medium in the left superior ophthalmic vein in the venous phase (Fig. 3A). Furthermore, angiography showed that the left internal carotid artery was narrowed in the arterial phase (Fig. 3B). External

Received July 25, 2011; Accepted October 4, 2011

Author's present address: H. Okamoto, MD, Department of Neurosurgery, Faculty of Medicine, Saga University, Saga, Saga, Japan. 

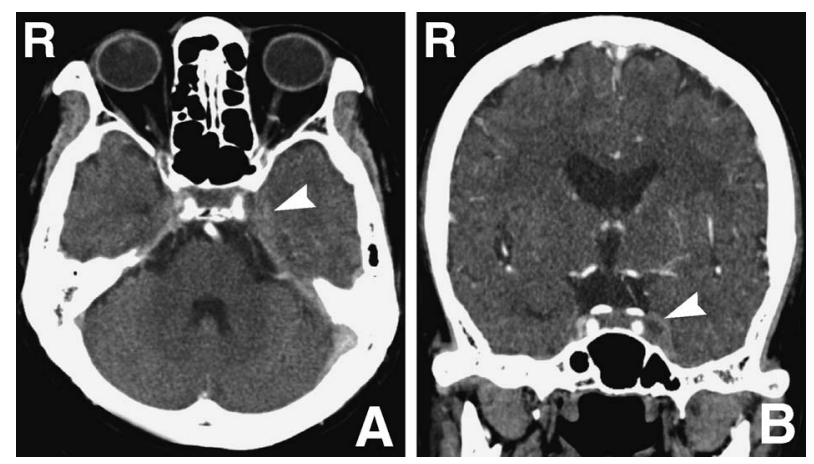

Fig. 1 Case 1. Axial (A) and coronal (B) computed tomography scans with contrast medium showing a filling defect in the left cavernous sinus (arrowheads).
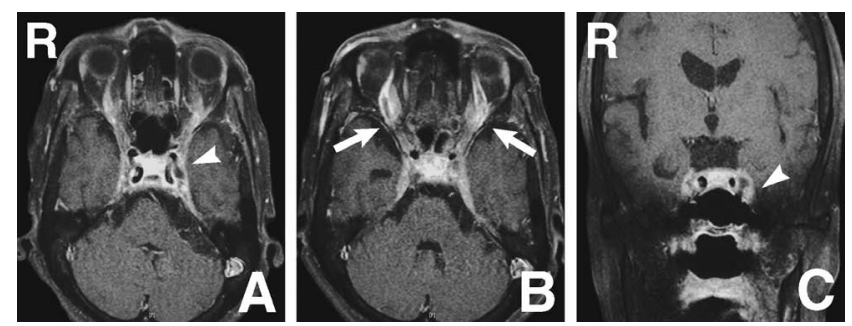

Fig. 2 Case 1. Axial (A, B) and coronal (C) $\mathrm{T}_{1}$-weighted magnetic resonance images with gadolinium showing a filling defect in the left cavernous sinus ( $A, C$, arrowheads), and venous dilation of the bilateral ophthalmic veins (B, arrows).
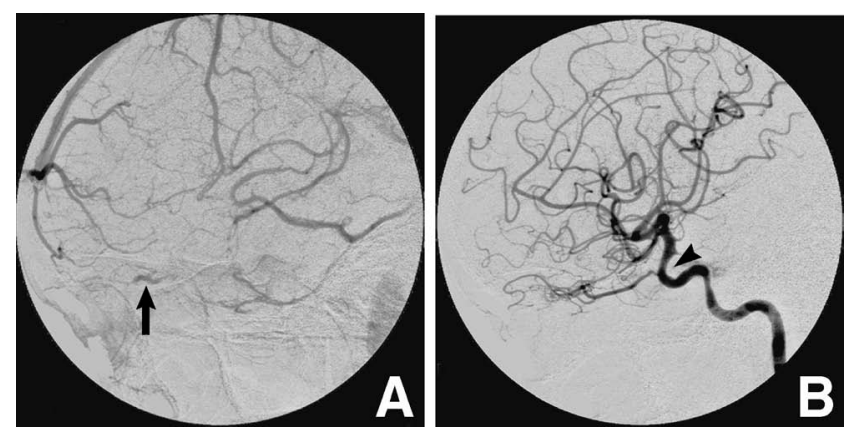

Fig. 3 Case 1. Left internal carotid angiograms (lateral view) showing a dilated ophthalmic vein in the venous phase (A, arrow) and narrowing change in the left internal carotid artery in the arterial phase (B, arrowhead).

carotid angiography showed no definite abnormality (data not shown).

We suspected CST associated with tooth extraction but could not distinguish it from other diseases related to cavernous sinus syndrome. CST requires immediate treatment, so we initially started intravenous broad-spectrum penicillin (ampicillin/sulbactam) on the 1st day of admission. Her headache decreased on the 2nd hospital day and fever also improved. Ptosis and eye movement improved from the 7 th hospital day. In addition, laboratory data
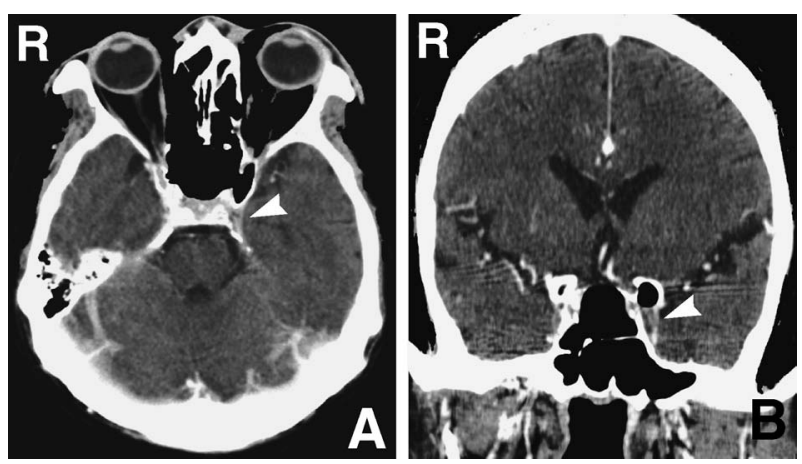

Fig. 4 Case 2. Axial (A) and coronal (B) computed tomography scans with contrast medium showing a filling defect in the left cavernous sinus (arrowheads).
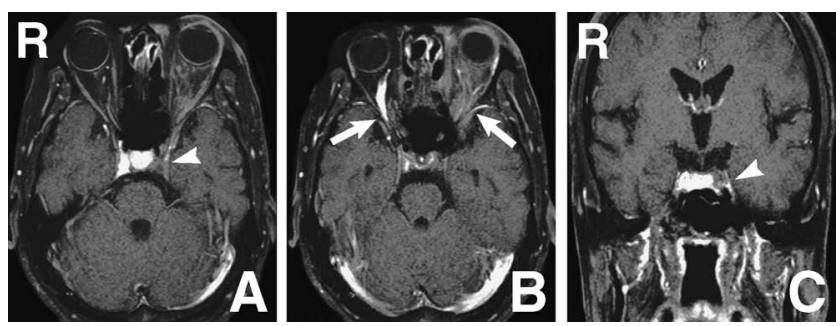

Fig. 5 Case 2. Axial (A, B) and coronal (C) $\mathrm{T}_{1}$-weighted magnetic resonance images with gadolinium showing a filling defect in the left cavernous sinus (A, C, arrowheads), and venous dilation of the bilateral ophthalmic veins with poor enhancement of the left ophthalmic vein (B, arrows).

showed steady improvement. We continued antibacterial therapy for 2 weeks until her body temperature and blood inflammation data normalized. Palsies of the left oculomotor and trochlear nerves had almost disappeared on the 14th hospital day.

Case 2: A 54-year-old man complained of pain in the left maxilla for 2 weeks. He was treated by a dentist for multiple dental caries and periodontitis including the teeth in the left maxilla. On the next day, he developed severe pain and swelling in the left forehead and face and was referred to an otolaryngologist who prescribed oral antibiotics and NSAID which were ineffective. Three days later, the patient developed periorbital swelling and pain. In addition, laboratory data showed a peripheral WBC count of $14000 / \mu \mathrm{l}$ and CRP level of $30.7 \mathrm{mg} / \mathrm{dl}$. The otolaryngologist suspected severe periorbital cellulitis and referred the patient to our hospital after treatment with carbapenems.

On admission to our hospital, ptosis, proptosis, and chemosis were observed in the left eye, but body temperature was within the normal range. The patient presented with swelling in the upper and middle area of the left face. Neurological examination on admission revealed palsy of the left trochlear nerve and hypesthesia in the ophthalmic and maxillary divisions of the left trigeminal nerve. Dental examination showed multiple caries and periodontitis but no abscess in the oral cavity. CSF from a lumbar puncture 
contained $1 \mathrm{WBC} / \mathrm{mm}^{3}$ with normal opening pressure. The protein level was elevated ( $94 \mathrm{mg} / \mathrm{dl}$ ); the glucose level was $165 \mathrm{mg} / \mathrm{dl}$ (blood glucose $360 \mathrm{mg} / \mathrm{dl}$ ). Blood and CSF culture findings were negative.

Head CT and MR imaging with contrast medium showed a non-enhanced area within the left cavernous sinus with dilation of the bilateral superior ophthalmic veins, similar to the observations in Case 1, and poor enhancement of the left ophthalmic vein (Figs. 4 and 5). These findings suggested obstruction of the cavernous sinus.

We suspected periorbital cellulitis followed by CST after dental infection and started treatment with carbapenems (meropenem), which had been previously administered by the otolaryngologist. Ptosis improved on the 2nd hospital day. Headache and swelling of the face decreased on the 4th hospital day, and left trochlear palsy improved on the 9th hospital day. We continued antibiotic administration for 2 weeks until blood inflammation data normalized. Pain and mild sensory disturbance around the left orbit remained when the patient was discharged from our hospital on the 21st hospital day.

\section{Discussion}

The diagnosis of CST is based on the clinical findings, characterized by various clinical manifestations, such as fever, pain, proptosis, chemosis, periorbital swelling, ophthalmoplegia, and sensory disturbances in the distribution of the ophthalmic and maxillary nerves. ${ }^{1,3,7,9,11)}$ The most consistent presentations in CST are fever, chemosis, proptosis, and periorbital edema (95\%), which are generally followed by extraocular muscle palsies $(88 \%))^{1,7,9)}$ Lateral gaze palsies appear initially and are the most severe because the sixth cranial nerve is most commonly affected. The sixth cranial nerve passes medially through the cavernous sinus, so may be susceptible to the inflammation in CST. Meanwhile, the third and fourth cranial nerves are less affected because they are located in the lateral region with thick fibrous tissue in the cavernous sinus. Approximately $20 \%$ of patients with CST present with dysfunction of the ophthalmic and maxillary nerves. Visual impairment including blindness is observed in $20 \%$ of patients with CST. If CST progresses, patients become lethargic or ultimately comatose.

These manifestations are often unique. Although CST requires prompt treatment, delay may occur because of confusion during the diagnosis. Therefore, CST should be considered first in the differential diagnosis of patients with cavernous sinus syndrome presenting with infectious symptoms such as fever, facial swelling, or abnormal laboratory data. CT and MR imaging with contrast medium can support the diagnosis of CST. ${ }^{1,3,7)}$ The direct signs of thrombosis in the cavernous sinus include expansion of the cavernous sinus, convex shaping of the lateral wall, and asymmetrical enhancement between each side. ${ }^{1)}$ An abnormal partial filling defect in the cavernous sinus caused by the thrombus can occasionally be observed. The indirect signs of thrombosis in the cavernous sinus reveal dilation of the superior ophthalmic veins or exoph- thalmos. ${ }^{1)}$

Cerebral angiography has been reported as not useful for the diagnosis of CST because even in normal studies, the cavernous sinus is often poorly visualized by carotid angiography. ${ }^{7,11)}$ Furthermore, some reports have warned of the risk of dissemination of infection and extension of thrombosis by angiography. ${ }^{1)}$ We performed cerebral angiography in Case 1 to exclude other distinguishing diseases such as carotid-cavernous sinus fistula. Abnormal findings of the internal carotid artery such as stenosis, occlusion, or aneurysm have been observed in patients with CST, 1,6,7,9) and angiography in Case 1 showed irregularity of the left internal carotid artery in the arterial phase, possibly indicating arteritis, spasm, or thrombosis.

The most common etiology of CST is sinusitis, particularly sphenoid and ethmoid sinusitis. ${ }^{1,7,9)}$ Complicated facial infection was one of the frequent causes of CST before the introduction of antibiotics, but recently has been less common. ${ }^{1)}$ The decrease in CST caused by complicated facial infections may be the reason for the current rarity of CST. Both of the present cases were assumed to be related to dental infection. CST caused by oral and dental infection is rarer than sinusitis and is identified in $10 \%$ or less of CST cases. ${ }^{1,2,12)}$ Oral bacteria from dental infection may spread to the cavernous sinus from the orbit through the pterygopalatine space or the pterygoid plexus or facial veins hematogenously. ${ }^{8,10)}$

The most important treatment for CST is administration of antibiotics that are sensitive to the pathogen. The most common pathogen in CST is Staphylococcus aureus. ${ }^{1,3)}$ In addition, beta-hemolytic streptococci, other rare streptococci, anaerobes, or mixed organisms are common organisms in patients with CST related to oral and dental infection. ${ }^{1,8)}$ Broad-spectrum penicillins, third-generation cephalosporins, or carbapenems are recommended for most etiologies and should be administered for at least 2 weeks to prevent any recurrence. ${ }^{1,7)}$ In our cases, blood and CSF culture findings were negative because antibiotics had previously been administered prior to admission.

Antibiotic therapy is essential, but induction of anticoagulant therapy has been disputed until now..$^{1,3,4,7-10)}$ Anticoagulant therapy may be effective for the prevention of the extension of thrombosis or dissemination of septic embolization, but may cause fatal systemic hemorrhage or intracranial hemorrhage (ICH). CST is rare, so only retrospective studies are available. Review of 84 cases reported between 1940 and 1984 found that the mortality rate in patients with heparin treatment was significantly lower than that in patients without heparin treatment (14\% versus $36 \%$, respectively) ${ }^{9)}$ Anticoagulant treatment may be safely administered for CST although a few cases of systemic hemorrhage or ICH have been reported.1 ${ }^{1}$ Fortunately, our patients recovered well without anticoagulation treatment, but it should be considered for future cases. Systemic administration of corticosteroids is controversial, but may be useful for the improvement of cranial nerve dysfunction or reduction of orbital congestion although risks acceleration of further thrombosis or worsening of infectious conditions. ${ }^{1,3,7,9)}$

CST recurrence after discontinuation of antibiotic ther- 
apy has been reported.1) Furthermore, intra-cavernous aneurysms occurred secondary to CST and such arterial changes appeared within 3 months after the development of initial symptoms. ${ }^{5}$ ) Therefore, patients with CST need follow up for at least several months after antibiotic treatment. Prompt diagnosis followed by correct treatment such as that with antibiotics is crucial for CST. CST is rare at present, but should be strongly considered as a possible underlying disease in patients with swelling of the face or cavernous sinus syndrome.

\section{Acknowledgment}

We are thankful to Ms. Barbara Ikejiri for her assistance in the preparation of this manuscript.

\section{References}

1) Bhatia K, Jones NS: Septic cavernous sinus thrombosis secondary to sinusitis: are anticoagulants indicated? A review of the literature. J Laryngol Otol 116: 667-676, 2002

2) Colbert S, Cameron M, Williams J: Septic thrombosis of the cavernous sinus and dental infection. Br J Oral Maxillofac Surg 49: e25-26, 2011

3) Ebright JR, Pace MT, Niazi AF: Septic thrombosis of the cavernous sinuses. Arch Intern Med 161: 2671-2676, 2001

4) Einhaupl KM, Villringer A, Meister W, Mehraein S, Garner C, Pellkofer M, Haberl RL, Pfister HW, Schmiedek P: Heparin treatment in sinus venous thrombosis. Lancet 338: 597-600, 1991
5) Endo S, Ohtsuji T, Fukuda O, Oka N, Takaku A: A case of septic cavernous sinus thrombosis with sequential dynamic angiographic changes. A case report. Surg Neurol 32: 59-63, 1989

6) Hoshino C, Satoh N, Sugawara S, Kuriyama C, Kikuchi A, Ohta M: Septic cavernous sinus thrombosis complicated by narrowing of the internal carotid artery, subarachnoid abscess and multiple pulmonary septic emboli. Intern Med 46: 317-323, 2007

7) Kriss TC, Kriss VM, Warf BC: Cavernous sinus thrombophlebitis: case report. Neurosurgery 39: 385-389, 1996

8) Ogundiya DA, Keith DA, Mirowski J: Cavernous sinus thrombosis and blindness as complications of an odontogenic infection: report of a case and review of literature. J Oral Maxillofac Surg 47: 1317-1321, 1989

9) Southwick FS, Richardson EP Jr, Swartz MN: Septic thrombosis of the dural venous sinuses. Medicine (Baltimore) 65: 82-106, 1986

10) Yarington CT Jr: Cavernous sinus thrombosis revisited. Proc R Soc Med 70: 456-459, 1977

11) Yasargil MG, Damur M: Thrombosis of the cerebral veins and dural sinuses, in Newton TH, Potts DG (eds): Radiology of the Skull and Brain, Angiography. St Louis, CV Mosby, 1981, pp 2375-2400

12) Yun MW, Hwang CF, Lui CC: Cavernous sinus thrombosis following odontogenic and cervicofacial infection. Eur Arch Otorhinolaryngol 248: 422-424, 1991

Address reprint requests to: Hiroaki Okamoto, MD, Department of Neurosurgery, Faculty of Medicine, Saga University, 5-1-1 Nabeshima, Saga, Saga 849-8501, Japan. 